\title{
A multi-objective location-inventory model for 3PL providers with sustainable considerations under uncertainty
}

\author{
R. Daghigh ${ }^{a^{*}}$, M.S. Jabalameli ${ }^{\mathrm{a}}$, A. Bozorgi Amiri ${ }^{\mathrm{b}}$ and M.S. Pishvaee ${ }^{\mathrm{a}}$
}

${ }^{a}$ Department of Industrial Engineering, Iran University of Science and Technology, Tehran, Iran ${ }^{b}$ School of Industrial Engineering, College of Engineering, University of Tehran, Tehran, Iran

CHRONICLE ABSTRACT

Article history:

Received November 42015

Received in Revised Format

December 212015

Accepted March 152016

Available online

March 152016

Keywords:

Sustainable Development

Supply chain network design

Multi-objective optimization

Possibilistic programming

\begin{abstract}
In recent years, logistics development is considered as an important aspect of any country's development. Outsourcing logistics activities to third party logistics (3PL) providers is a common way to achieve logistics development. On the other hand, globalization and increasing customers' concern about the environmental impact of activities as well as the appearance of the issue of social responsibility have led companies employ sustainable supply chain management, which considers economic, environmental and social benefits, simultaneously. This paper proposes a multi-objective model to design logistics network for 3PL providers by considering sustainable objectives under uncertainty. Objective functions include minimizing the total cost, minimizing greenhouse gas emission and maximizing social responsibility subject to fair access to products, number of created job opportunities and local community development. It is worth mentioning that in the present paper the perishability of products is also considered. A numerical example is provided to solve and validate model using augmented Epsilon-Constraint method. The results show that three sustainable objectives were in conflict and as the one receives more desirable values, the others fall into more undesirable values. In addition, by increasing maximum perishable time periods and by considering lateral transshipment among facilities of a level one can improve sustainability indices of the problem, which indicates the necessity of such policy in improving network sustainability.
\end{abstract}

\section{Introduction}

As the experience of almost four decades of pioneer countries and industries shows: effectiveness of logistics and supply chain is one of the most important approaches to improve businesses and reduce transaction costs. Today, it is essential for industry and business managers to pay more attention to logistics, since it constitutes up to 30 percent of delivery costs of the products. Therefore, it is important to promote the knowledge of logistics to create the third party logistics companies and their increasing global appeals. Third party logistics companies, abbreviated as 3PL, are the firms to which production or service companies outsource their logistics issues, partially or completely (Boyson et al., 1999). However, globalization, increase of governmental and non-governmental regulations, and the pressure

* Corresponding author.

E-mail: rozitadaghigh@yahoo.com (R. Daghigh)

(C) 2016 Growing Science Ltd. All rights reserved. doi: $10.5267 /$ j. ijiec.2016.3.003 
and demand of clients regarding environmental issues as well as advent of social responsibility of companies, have motivated organizations to study the required steps of implementing sustainable supply chain management in order to improve environmental, social, and economical performance. Since sustainable development of a country depends on maintenance and optimum utilization of limited and irreplaceable resources in that country, various actions are considered by governments regarding this issue, which includes using raw material compatible with environment in production and industrial centers, reducing usage of fossil and oil energy sources, and reusing waste products (Pishvaee et al., 2014). Furthermore, governments have passed rules to support environment, such as greenhouse gas reduction regulation in European Union, Australia, and Canada. Complicated and dynamic nature of supply chains inject a high degree of uncertainty into decisions which is an inevitable feature of any supply chain. Generally, uncertainty in data can be categorized in two groups (Mula et al., 2006): 1Randomness, which is the result of inherent randomness of the parameters. 2- Epistemic uncertainty, which deals with insufficient knowledge and inaccurate parameters resulted from knowledge considering their accurate value.

Considering the input data of the problem, strategic design of forward logistics network for third party logistics providers is executed in this paper regarding sustainability considerations under uncertain environment aiming to effectively manage 3PLS possible processes. In this 4-stage network, products are collected from various producers to meet demands and are transshipped to Cross Docks. In cross docks, received shipments from several producers are aggregated and combined together and after being sorted in trucks they are sent to distribution centers via large size dispatching in order to benefit from economy of scale.

In order to moderate demand variations and prevent from facing shortage, lateral transshipment technique and inventory are used among distribution centers and transshipped to customer regions. Moreover, in addition to objective function of cost, sustainability issues are also considered as extra objective in the model. Uncertain conditions in this problem are under the influence of costs and demands and some input parameters of the problem and inaccurate parameters are involved by triangular possibilistic distributions and in order to control uncertain conditions in the parameters, possibilistic programming is used. This approach stabilizes model results against input parameter fluctuations and minimizes its dispersion.

The rest of the paper is organized as follows: in section 2 a literature review is conducted. In section 3 , the problem and the modeling are described. In section 4, the proposed approach for solving the possibilistic multi-objective problem is presented. In section 5, a numerical example is solved and sensitivity analysis has been performed in order to validate the model. Finally in section 6 , conclusions and suggestions for future study are presented.

\section{Literature review}

During the past few years, several studies regarding logistics network design have been conducted to fit this problem into real-world circumstances. However, since outsourcing logistics activities to third party logistics companies, is a relatively new context, few works have been accomplished regarding logistics network design. Ko and Evans (2007) presented a network design model for third party logistics company. They investigated forward and reverse logistics movement using dynamic parameters. Then they used simulation technique to study uncertainty into their model, and the model was solved using hybrid genetic algorithm. Zhang et al. (2007) presented a fuzzy model to design a network for reproduction logistics, from 3PL point of view. This model was under reverse logistics process. They used fuzzy chance constrained programming model in network design problem. In this paper, transportation costs and parameters related to backward demand of products were considered as triangular fuzzy numbers. Mallidis et al. (2012) presented a green supply chain network design model for the first time which included input ports, distribution centers, and transportation modes along with 
decisions about dedicated or shared usage of storehouses (3PL) and transportation vehicles considering environmental effects of transportation. Ghaffari-Nasab et al. (2015) proposed a hub logistics network design from the perspective of third party companies by considering inventory costs and the existing uncertainty in demand regarding production and distribution.

Today, it is tried to optimize problems from different aspects, since, dealing with only one aspect can neutralize other essential aspects in decisions. However, by considering the increasing importance of sustainability, many studies have chosen environmental or social effects as extra goals in designing multiobjective sustainable supply chain network. Dehghanian and Mansour (2009) developed a mathematical programming model including three objective functions to increase economic aspect and social benefit and reduce environmental effects. The aim of this paper was to design a sustainable recycling network to balance all three sustainability factors and life cycle analysis (LCA) was used to study environmental effects of various End-of-Life options. Multi-objective genetic algorithm was implemented to find Pareto optimal solutions and the study was implemented for rubber wastes. Pishvaee et al. (2014) proposed a multi-objective possibilistic programming model to design sustainable medical supply chain by considering economic, environmental and social objective functions under uncertainty conditions. In order to solve this proposed model, Benders decomposition algorithm was also implemented.

Devika et al. (2014) developed a mix integer programming model for multi-objective closed-loop supply chain network to take into account all three sustainability factors in network design, simultaneously. In order to solve this complicated problem, three hybrid metaheuristics, which are based on imperialistic competitive algorithm and variable neighbor search algorithm, were utilized. Finally, glass industry case study was used to show the application of this approach. Ramezani et al. (2014) demonstrated fuzzy set application in designing multi-period closed-loop supply chain network having multiple products. The presented model includes three objective functions: profit increase, delivery time reduction, and quality increase. Using fuzzy approach, flexible restrictions, and fuzzy coefficients, an efficient model was obtained.

As it can be inferred from mentioned researches, third party logistics network design problems and sustainable network design are amongst important subjects for research; however, almost no model can be found that has uniformly considered these issues. Therefore, developing third party logistics network design models which consider sustainability is an appealing research subject and is of high importance. Furthermore, since there are perishable products in real world, no model has been seen that considers these conditions in both third party logistics network design and sustainability fields.

The aim of this paper is to present a multi-period multi-objective possibilistic programming model with several transportation modes for perishable products having a lateral transship among facilities of a level in order to design sustainable network of third party logistics providers. The first goal of this problem is to minimize total cost of the system and the second one is to maximize the social profits. The second goal is due to the fact that from the company directors' perspective, social responsibility can improve social image and brand of the company and reduce risk. The primary duty of 3PL companies is to transport goods from producers to applicants by having reaching customer satisfaction and they are responsible for organizing the majority of markets, which shows the importance of social performance of the company in forms of justly responsibility and serviceability for all customer regions at any period of time. For this purpose, in problem modeling, the maximum demands not met for any type of product at any period and for any customer region is minimized, so that the primary duty, which is distribution and availability of products for customer regions, is accomplished. Moreover, in addition to fair distribution, the company aims to create more job opportunities through building cross docks and distribution centers in areas with high unemployment rate. It has also aimed to create economic development balance through focusing more on areas with low economic development. However, since transportation is one the main operations of 3PL companies, it has the most influence in increasing 
greenhouse gases. The third goal is to minimize the emission of greenhouse gases from various transportation vehicles.

The most important innovations and considerations that distinguish this paper from other papers in this field are as follows:

1. Simultaneous consideration of third party logistics network design issues and environmental issues.

2. Considering social aspect of sustainability suitable for application of problem in network design problem of the third party logistics companies.

3. Considering perishable of products in network design of third party logistics providers.

4. Presenting an integrated multi-objective multi-period inventory-locating model with multiple transportation modes for perishable products accompanied with lateral transship among facilities of a level in uncertain conditions.

5. Using possibilistic programming model to oppose the existing uncertainty in some inputs of the Problem description.

\section{Problem description}

The network under study is a 4-level network, which comprises of producers set, $I$, candidate locations for locating cross docks set, $J$, distribution centers set, $K$, and customer regions set, $R$. In this network, third party logistics provider company (3PL) is responsible for managing logistics activities concerning product flow management for multiple customers (producers). The company collects products from various producers and ships them to cross docks. In cross docks, received shipments from several producers are combined together and after sorting and aggregation of the flow, products are shipped to distribution centers as soon as possible. Products are held in distribution centers to moderate demand variations, due to proximity to customer regions. These products are then shipped to customer regions from distribution centers. Fig. 1 illustrates the schematic of the whole network.

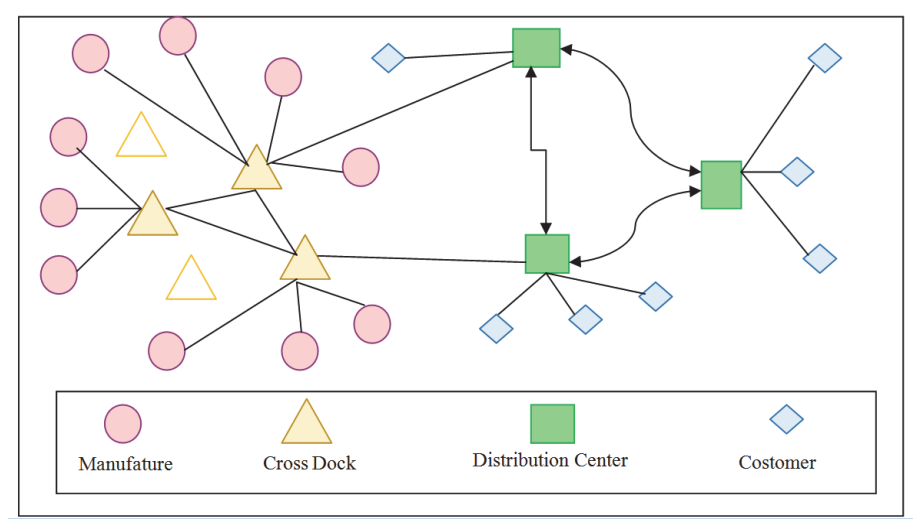

Fig. 1. Logistics network for a 3PL firm

In this network, the 3PL company has used complete lateral transshipment technique in order to prevent facing shortage or surplus predicted demand and reducing transportation cost (Torabi \& Moghaddam, 2013).

\subsection{Model assumptions}

1. Due to dynamic nature of business environment in which third party logistics providers operate, the model is multi-period and the network structure is determined for each cycle. 
2. Production center and customer region locations are predetermined and locating decisions are regarding cross docks and select decisions are regarding distribution centers.

3. Each producer only produces its unique product and capacity restrictions are considered for production site.

4. Several products flow in this network and since cross docks are appropriate and beneficial for perishable products, perishable assumption for products is considered, which are from perishable type with fixed shelf life-time (possessing maximum consumable life).

5. 3PLs hold and distribute products via renting distribution centers, the price of which is dependent on the location and capacity of the center. Some capacity restrictions are also considered for distribution centers.

6. It is assumed that inventory distribution in any distribution center at the end of each period becomes zero, because that distribution center may not be selected for the next period.

7. In this network, each producer sends its products to one cross dock only and each distribution center is also allocated to one cross dock (singular allocation feature). This assumption is more economic, because it will cause larger stacks to be sent to one cross dock than the case where smaller stacks are sent to multiple cross docks.

8. There are different modes of transportation vehicles, each having various capacities, environmental effects and transportation costs.

9. Problem parameters such as transportation costs between supply chain levels, lateral transshipment among distribution centers, cost of inventory in each period, locating and operating cross docks in each period, selecting and hiring distribution centers, shortage costs, and demands are uncertain.

\subsection{Indices, parameters, and variables}

Notations used in the model are presented below. Uncertain parameters of the proposed model are specified with tilde symbol. Then the mathematical model is developed under uncertain conditions.

Indices

I Index for manufacturing facilities $i=\{1,2, \ldots, I\}$

$J \quad$ Index for potential Cross docks $j=\{1,2, \ldots J\}$

$K \quad$ Index for distribution centers $k=\{1,2, \ldots, K\}$

$W \quad$ Index fordifferent size of distribution centers $w=\{1,2, \ldots, W\}$

$R \quad$ Index for customer zones $r=\{1,2, \ldots, R\}$

$P \quad$ Index for different products $p=\{1, \ldots, P\}$

$M \quad$ Index for transportation modes $m=\{1,2, \ldots, M\}$

$T \quad$ Index for time periods $t=\{1,2, \ldots, T\}$

\section{Parameters}

$F \tilde{W}_{j t} \quad$ Fixed cost of establishing a cross dock $j$ in time period $t$

$F \tilde{S}_{j t} \quad$ Performance cost of a cross dock $j$ in time period $t$

$F \tilde{K}_{k w t} \quad$ Leasing cost of a distribution center $k$ with size $w$ in time period $t$

$\tilde{C}_{i j m} \quad$ Transportation cost from manufacturer $i$ to cross dock $j$ with transportation mode $m$

$\theta$ discount factor representing the economy of scale for consolidated shipments between cross docks

$\tilde{C} T_{j l m} \quad$ Transportation cost from cross dock $j$ to cross dock $l$ with transportation mode $m$ 
$C \tilde{P}_{l k m} \quad$ Transportation cost from cross dock $l$ to distribution center $k$ with transportation mode $m$

$C \tilde{O}_{k r m} \quad$ Transportation cost from distribution center $k$ to customer $r$ with transportation mode $m$

$\operatorname{COS} \tilde{T}_{k k^{\prime} m}$ Transshipment cost from distribution center $k$ to distribution center $k^{\prime}$ with transportation mode $m$

$H \tilde{I}_{k p t} \quad$ inventory holding cost of product $p$ at distribution center $k$ in time period $t$

$H l \tilde{s}_{k p t} \quad$ Lost sale cost of product $p$ at distribution center $k$ in period $t$

$d \tilde{e}_{p r t} \quad$ Demand of customer $r$ in time period $t$

$l a_{k w} \quad$ Capacity of a distribution center $k$ of size $w$

$\operatorname{cap}_{m} \quad$ Capacity of transportation mode $m$

$m m_{p i} \quad$ Capacity of manufacturer $i$ for product $p$

$t \max _{p} \quad$ Maximum consecutive time periods that a perishable product $p$ can be stored

$d_{i j} \quad$ distance between nodes $i$ and $j$

$d_{j l} \quad$ distance between nodes $j$ and $l$

$d_{l k} \quad$ distance between nodes $l$ and $k$

$d_{k k^{\prime}} \quad$ distance between nodes $k$ and $k^{\prime}$

$d_{k r} \quad$ distance between nodes $k$ and $r$

$\mu \quad$ a large value number

$\omega^{\mathrm{m}} \quad$ Curb-weight of transportation mode $m$

$n_{t f}{ }^{m} \quad$ Vehicle drive train efficiency for transportation mode $m$

$\eta, \kappa \quad$ Constant value

$\xi \quad$ Fuel-to-air mass ratio

$K^{m} \quad$ Engine friction factor for transportation mode $m$

$N^{m} \quad$ Engine speed for transportation mode $m$

$v d^{m} \quad$ Engine displacement of transportation $m$

$\propto \quad$ Acceleration of transportation mode $m$

$g \quad$ Gravitational constant

$\varphi_{i j} \quad$ road grade angle in degrees,

$c_{r}{ }^{m} \quad$ Coefficient of rolling resistance of transportation mode $m$

$c_{d}{ }^{m} \quad$ Coefficient of aerodynamic drag of transportation mode $m$

$A^{m} \quad$ Frontal surface area of transportation mode $m$

$\rho \quad$ Air density

$v_{i j}{ }^{m} \quad$ Speed of transportation mode $m$ between node $i$ and node $j$

$v_{j l}{ }^{m} \quad$ Speed of transportation mode $m$ between node $j$ and node $l$

$v_{l k}{ }^{m} \quad$ Speed of transportation mode $m$ between node $l$ and node $k$

$v_{k r}{ }^{m} \quad$ Speed of transportation mode $m$ between node $k$ and node $r$

$v_{k k^{\prime}}{ }^{m} \quad$ Speed of transportation mode $m$ between node $k$ and node $k^{\prime}$

$s^{m} \quad$ The greenhouse gas emission index coefficient for transportation mode $m$

$j c \tilde{c}_{j} \quad$ number of created job opportunities if a cross dock is opened at location $j$

$j d \tilde{c}_{k}^{w} \quad$ number of created job opportunities if a distribution center is opened at location $\mathrm{k}$ with size $w$ 
$u_{j} \quad$ unemployment rate at location $j$

$u_{k} \quad$ unemployment rate at location $k$

$v \tilde{p}_{j} \quad$ economic value of cross dock $j$

$v \tilde{p}_{k}^{w} \quad$ economic value of distribution center $k$ with size $w$

$e d c_{j} \quad$ level of regional development at location $j$

$e d c_{k} \quad$ level of regional development at location $k$

$s \tilde{i}_{\max }^{m m x} \quad$ maximum possible value of social impact related to Equitable access subcategories

$s \tilde{i}_{\min }^{m m x} \quad$ minimum possible value of social impact related to Equitable access subcategory

$s \tilde{i}_{\max }^{j c} \quad$ maximum possible value of social impact related to "employment" subcategories

$s \tilde{i}_{\min }^{j c} \quad$ minimum possible value of social impact related to "employment" subcategories

$s \tilde{i}_{\max }^{p t} \quad$ Maximum possible value of social impact related to "balanced economic development" subcategory

$s \tilde{i}_{\min }^{p t} \quad$ minimum possible value of social impact related to "balanced economic development" subcategory

$w p \quad$ importance weight of social impact indicator related to Equitable access subcategory

$w c \quad$ importance weight of social impact indicator related to employment subcategory

wt importance weight of social impact indicator related to (balanced) economic development subcategory

\section{Continues variables}

$X_{\text {pijmt }} \quad$ The amount of product $p$ shipped from manufacturer $i$ to cross dock $j$ by transportation mode $m$ in time period $t$.

$O_{p j l m t} \quad$ The amount of product $p$ shipped from cross dock $j$ to cross dock $l$ by transportation mode $m$ in time period $t$.

$\pi_{p l k m t} \quad$ The amount of product $p$ shipped from cross $\operatorname{dock} l$ to distribution center $k$ by transportation mode $m$ in time period $t$.

$H_{p k r m t} \quad$ The amount of product $p$ shipped from distribution center $k$ to customer zone $r$ by transportation mode $m$ in time period $t$.

$V T_{p k k^{\prime} m t} \quad$ The amount of product $p$ shipped from distribution center $k$ to distribution center $k^{\prime}$ by transportation mode $m$ in time period $t$.

$S_{p k r m t_{1} t} \quad$ The amount of product $p$ produced in time period $t_{1}$ and shipped from distribution center $k$ to customer zone $r$ by transportation mode $m$ in time period $t$.

$T R_{k k^{\prime} p m t 1 t} \quad$ The amount of product $p$ produced in time period $t_{1}$ and shipped from distribution center $k$ to distribution center $k^{\prime}$ by transportation mode $m$ in time period $t$.

$I^{\prime}{ }_{p k t_{1} t} \quad$ inventory of product $p$ produced in time period $t_{1}$ in distribution center $k$ in period $t$

$I_{p k t} \quad$ inventory of product $p$ produced in distribution center $k$ in period $t$

$l s_{p r t} \quad$ shortage of product $p$ produced in customer zone $r$ in period $t$

Binary variables

$Z_{j t}\left\{\begin{array}{l}1 \text { if a cross dock is opened at location } j \text { in time period } t \\ 0 \quad \text { otherwise }\end{array}\right.$ 
$V d c_{k t}^{w}\left\{\begin{array}{l}1 \text { if a distribution center with size } w \text { is opened at location } k \text { in time period } t \\ 0 \quad \text { otherwise }\end{array}\right.$

$W_{i j t}\left\{\begin{array}{c}1 \text { if a manufaturer } i \text { to a cross dock } j \text { is allocate in time period } t \\ 0 \quad \text { otherwise }\end{array}\right.$ $Y_{k l t}\left\{\begin{array}{c}1 \text { if a distribution center } k \text { to a cross dock } j \text { is allocate in time period } t \\ 0 \quad \text { otherwise }\end{array}\right.$

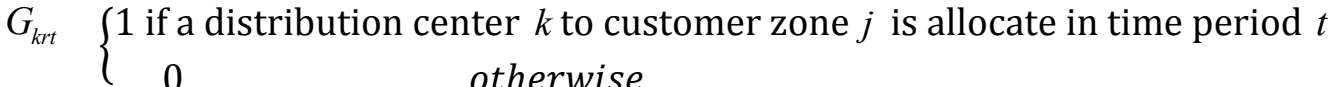
tras $^{\prime}{ }_{j l t} \quad\left\{\begin{array}{c}1 \text { if a cross dock } j \text { a cross dock } l \text { is allocate in time period } t \\ 0 \quad \text { otherwise }\end{array}\right.$

$\operatorname{tras}^{i i}{ }_{k k^{\prime} t}\left\{\begin{array}{c}1 \text { if a distribution center } k \text { to distribution } k^{\prime} \text { is allocate in time period } t \\ 0 \quad \text { otherwise }\end{array}\right.$

$F x_{i j m t}\left\{\begin{array}{c}1 \text { if a transportation mode } m \text { traverses node } i \text { to node } j \text { in time period } t \\ 0 \quad \text { otherwise }\end{array}\right.$

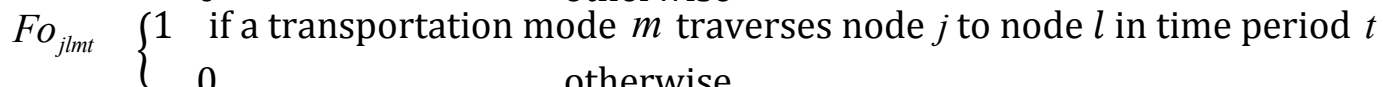

$F \pi_{l k m t} \quad\{1$ if a transportation mode $m$ traverses node $l$ to node $k$ in time period $t$

$F h_{k r m t}\left\{\begin{array}{c}1 \text { if a transportation mode } m \text { traverses node } k \text { to node } r \text { in time period } t \\ 0 \quad \text { otherwise }\end{array}\right.$

$F T_{k k^{\prime} m t}\left\{\begin{array}{cc}1 & \text { if a transportation mode } m \text { traverses node } k \text { to node } k^{\prime} \text { in time period } t \\ 0 & \text { otherwise }\end{array}\right.$

Positive integer variables

$n u m I_{m t}^{i j} \quad$ number of transportation mode $m$ is used from node $i$ to $j$ in time period $t$ num $/ I_{m t}^{j l}$ number of transportation mode $m$ is used from node $j$ to $l$ in time period $t$ num $/ I I_{m t}^{l k}$ number of transportation mode $m$ is used from node $l$ to $k$ in time period $t$ $n u m / V_{m t}^{k k^{\prime}} \quad$ number of transportation mode $m$ is used from node $k$ to $k^{\prime}$ in time period $t$ $n u m V_{m t}^{k r} \quad$ number of transportation mode $m$ is used from node $k$ to $r$ in time period $t$

\subsection{Mathematical model}

The three-objective mathematical model presented in this model is in the form of linear programing of a mix integer programing and considering the above parameters and variables, the following is presented.

$$
\begin{aligned}
& \min Z_{1}: \sum_{j} \sum_{t} F \tilde{W}_{j t} Z_{j t}\left(1-Z_{j t-1}\right)+\sum_{j} \sum_{t} F \tilde{s}_{j t} Z_{j t}+\sum_{k} \sum_{w} \sum_{t} F \tilde{K}_{k w} V d c_{k t}^{w}+ \\
& \sum_{p} \sum_{i} \sum_{j} \sum_{m} \sum_{t} \tilde{C}_{i j m} X_{p i j m t}+\sum_{p} \sum_{j} \sum_{l} \sum_{m} \sum_{t} \theta \tilde{C} T_{j l m} O_{p j l m t}+ \\
& +\sum_{p} \sum_{l} \sum_{k} \sum_{m} \sum_{t} C \tilde{P}_{l k m} \pi_{p l k m t}+\sum_{p} \sum_{k} \sum_{r} \sum_{m} \sum_{t} C \tilde{O}_{k r m} H_{p k r m t} \\
& +\sum_{p} \sum_{k^{\prime}} \sum_{k} \sum_{m} \sum_{t} C O S \tilde{T}_{k k^{\prime} m} V T_{p k k^{\prime} m t}+\sum_{p} \sum_{k} \sum_{t} H \tilde{I}_{p k t} I_{p k t}+\sum_{p} \sum_{k} \sum_{t} H l \tilde{s}_{p k t} l s_{p k t}
\end{aligned}
$$


$\operatorname{maxZ} Z_{2}: \mathrm{wp} \cdot \frac{s \tilde{i}_{\max }^{m m x}-\max _{p r t}\left(\widetilde{d e}_{p r t}-\sum_{k} \sum_{m} X D C_{p k r m t}\right)}{s \tilde{i}_{\max }^{m m x}-s \tilde{i}_{\min }^{m m x}}+$

$w c . \frac{\sum_{j} \sum_{t} Z_{j t} j c \tilde{c}_{j} u p_{j}+\sum_{k} \sum_{w} \sum_{t} V d c_{k t}^{w} j d \tilde{c}_{k}^{w} u p_{k}-s i_{\min }^{j c}}{s \tilde{i}_{\max }^{j c}-s \tilde{i}_{\min }^{j c}}+$

$w t . \frac{\sum_{j} \sum_{t} Z_{j t} v \tilde{p}_{j}\left(1-e d c_{j}\right)+\sum_{k} \sum_{w} \sum_{t} V d c_{k t}^{w} v \tilde{p}_{k}^{w}\left(1-e d c_{k}\right)-s \tilde{i}_{\min }^{p t}}{s \tilde{i}_{\max }^{p t}-s \tilde{i}_{\text {min }}^{p t}}$

$\operatorname{minZ}_{3}: \sum_{i} \sum_{j} \sum_{m} \sum_{t} \delta^{m} n u m l_{m t}^{i j}\left(K^{m} N^{m} V d^{m} \gamma^{m} \frac{d_{i j}}{v_{i j}^{m}}+\omega^{m} \Gamma^{m} a_{i j}^{m} d_{i j} \gamma^{m}+\Gamma^{m} v_{i j}^{m 2} \beta^{m} d_{i j} \gamma^{m}\right)+$

$\sum_{p} \sum_{i} \sum_{j} \sum_{m} \sum_{t} \delta^{m} \Gamma^{m} a_{i j}^{m} d_{i j} \gamma^{m} X_{p i j m t}$

$\sum_{j} \sum_{l} \sum_{m} \sum_{t} \delta^{m} n u m / I_{m t}^{j l}\left(K^{m} N^{m} V d^{m} \gamma^{m} \frac{d_{j l}}{v_{j l}^{m}}+\omega^{m} \Gamma^{m} a_{j l}^{m} d_{j l} \gamma^{m}+\Gamma^{m} v_{j l}^{m 2} \beta^{m} d_{j l} \gamma^{m}\right)+$

$\sum_{p} \sum_{j} \sum_{l} \sum_{m} \sum_{t} \delta^{m} \Gamma^{m} a_{j l}^{m} d_{j l} \gamma^{m} O_{p j l m t}+$

$\sum_{l} \sum_{k} \sum_{m} \sum_{t} \delta^{m} n u m / I I_{m t}^{l k}\left(K^{m} N^{m} V d^{m} \gamma^{m} \frac{d_{l k}}{v_{l k}^{m}}+\omega^{m} \Gamma^{m} a_{l k}^{m} d_{l k} \gamma^{m}+\Gamma^{m} v_{l k}^{m 2} \beta^{m} d_{l k} \gamma^{m}\right)+$

$\sum_{p} \sum_{l} \sum_{k} \sum_{m} \sum_{t} \delta^{m} \Gamma^{m} a_{l k}^{m} d_{l k} \gamma^{m} \pi_{p l k m t}+$

$\sum_{k} \sum_{r} \sum_{m} \sum_{t} \delta^{m} n u m / V_{m t}^{k k^{\prime}}\left(K^{m} N^{m} V d^{m} \gamma^{m} \frac{d_{k r}}{v_{k r}^{m}}+\omega^{m} \Gamma^{m} a_{k r}^{m} d_{k r} \gamma^{m}+\Gamma^{m} v_{k r}^{m 2} \beta^{m} d_{k r} \gamma^{m}\right)+$

$\sum_{p} \sum_{k} \sum_{r} \sum_{m} \sum_{t} \delta^{m} \Gamma^{m} a_{k r}^{m} d_{k r} \gamma^{m} H_{p k r m t}+$

$\sum_{k} \sum_{k^{\prime}} \sum_{m} \sum_{t} \delta^{m} n u m V_{m t}^{k r}\left(K^{m} N^{m} V d^{m} \gamma^{m} \frac{d_{k k^{\prime}}}{v_{k k^{\prime}}^{m}}+\omega^{m} \Gamma^{m} a_{k k^{\prime}}^{m} d_{k k^{\prime}} \cdot \gamma^{m}+\Gamma^{m} v_{k k^{\prime}}{ }^{2} \beta^{m} d_{k k^{\prime}} \cdot \gamma^{m}\right)+$

$\sum_{p} \sum_{k} \sum_{k^{\prime}} \sum_{m} \sum_{t} \delta^{m} \Gamma^{m} a_{k k^{\prime}}^{m} d_{k k^{\prime}} \cdot \gamma^{m} V T_{p k k^{\prime} m t}$

Location and allocation constraints

$$
\begin{aligned}
& \sum_{i} W_{i j t} \leq Z_{j t} \forall j, t \\
& \sum_{k} Y_{k l t} \leq Z_{l t} \forall l, t \\
& \sum_{l} Y_{k l t} \leq \sum_{w} V d c_{k t}^{w} \forall k, \mathrm{t} \\
& \sum_{l} \operatorname{tras}^{\prime}{ }_{j l t} \leq Z_{j t} \forall j, t \\
& \sum_{j} \operatorname{tras}^{\prime}{ }_{j l t} \leq Z_{l t} \forall l, t \\
& \sum_{k^{\prime}} \operatorname{tras}^{i \prime}{ }_{k k^{\prime} t} \leq \sum_{w} V d c_{k w t} \forall k, \mathrm{t} \\
& \sum_{k} \operatorname{tras}^{i{ }^{\prime \prime}}{ }_{k k^{\prime} t} \leq \sum_{w} V d c_{k^{\prime} w t} \forall k^{\prime}, \mathrm{t}
\end{aligned}
$$


624

$\sum_{r} G_{k r t} \leq \sum_{w} V d c_{k w t} \forall k, \mathrm{t}$

Single allocation constraints

$$
\begin{aligned}
& \sum_{j} W_{i j t} \leq 1 \forall i, \mathrm{t} \\
& \sum_{l} Y_{k l t} \leq 1 \quad \forall k, t \\
& \sum_{k} G_{k r t} \leq 1 \forall r, t
\end{aligned}
$$

Capacity constraints

$\sum_{p} I_{p k t} \leq \sum_{w} l a_{k w} V d c_{k t}^{w} \forall k, t$
$\sum_{j} \sum_{m} X_{p i j m t} \leq M M_{p i t} \forall i, \mathrm{t}$

Shortage constraint

$\sum_{k} \sum_{m} H_{p k r m t}+l s_{p r t} \geq d \tilde{e}_{p r t} \forall p, r, t$

Location constraints

$Z_{j t-1} \leq Z_{j t} \forall j, t$

$\sum_{w} V d c_{k t}^{w} \leq 1 \forall k, t$

Flow and allocation constraints

$\sum_{p} \sum_{m} X_{p i j m t} \leq \mu W_{i j t} \quad \forall i, j, t$
$\sum_{p} \sum_{m} \Pi_{p l k m t} \leq \mu Y_{k l t} \quad \forall l, k, t$

$\sum_{p} \sum_{m} H_{p k r m t} \leq \mu G_{k r t} \forall k, r, t$

$\sum_{p} \sum_{m} O_{p j l m t} \leq \mu \operatorname{tras}^{i}{ }_{j l t} \forall j, l, t$

$\sum_{p} \sum_{m} V T_{p k k^{\prime} m t} \leq \operatorname{tras}^{i \prime}{ }_{k k^{\prime} t} \forall k, k^{\prime}, t$

Flow balance constraint

$\sum_{i} \sum_{m} X_{p i j m t}+\sum_{l} \sum_{m} O_{p l j m t}=\sum_{k} \sum_{m} \pi_{p l k n t}+\sum_{l} \sum_{m} O_{p j l m t} \forall p, j, t$

Inventory constraints

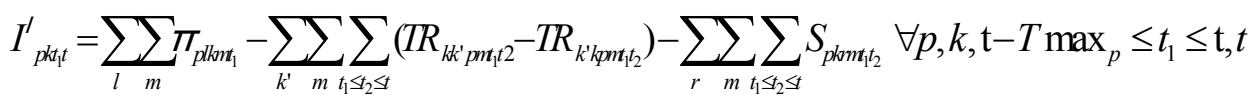




$$
\begin{aligned}
& I_{p k t}=\sum_{t_{1} \leq t} I_{p k t_{1} t}^{\prime} \forall p, k, t \\
& \sum_{t_{1} \leq t} S_{p k r m t_{1} t} \leq H_{p k r m t} \forall p, k, r, \mathrm{~m}, t \\
& \sum_{t_{1} \leq t} T R_{p k K^{\prime} m t 1 t} \leq V T_{p k K^{\prime} m t} \forall p, k, k^{\prime}, \mathrm{m}, t \\
& \sum_{k} \sum_{k^{\prime}} \sum_{m}\left(T R_{p k k^{\prime} m t_{1} t}+T R_{p k^{\prime} k m t_{1} t}\right)+\sum_{k} \sum_{r} \sum_{m} S_{p k r m t_{1} t}=0 \quad \forall p, t_{1} \prec t-T \max _{p}, t \\
& \sum_{k} I_{p k t_{1} t}=0 \quad \forall p, t_{1} \prec t-T \max _{p}, t \\
& \sum_{p} I_{p k t-1} \leq \mu \sum_{w} V d c_{k w}^{t} \forall k, t
\end{aligned}
$$

Relationship between flow and allocation of vehicles constraints

$$
\begin{array}{ll}
X_{p i j m t} \leq \mu F x_{i j m t} & \forall p, i, j, m, t \\
O_{p j l m t} \leq \mu F O_{j l m t} & \forall p, j, l, m, t \\
\Pi_{p l k m t} \leq \mu F \Pi_{l k m t} & \forall p, l, k, m, t \\
H_{p k r m t} \leq \mu F h_{k r m t} & \forall p, k, r, m, t \\
V T_{p k k^{\prime} m t} \leq \mu F T_{k k^{\prime} m t} & \forall p, k, k^{\prime}, m, t
\end{array}
$$

Capacity vehicles constraints

$$
\begin{aligned}
& \frac{\sum_{p} X_{p i j m t}}{\operatorname{cap}_{m}} \leq n u m l_{m t}^{i j} \leq \frac{\sum_{p} X_{p i j m t}}{\operatorname{cap}_{m}}+1 \forall i, j, m, t \\
& \frac{\sum_{p} O_{p j l m t}}{c a p_{m}} \leq n u m \|_{m t}^{j l} \leq \frac{\sum_{p} O_{p j l m t}}{c a p_{m}}+1 \forall j, l, m, t \\
& \frac{\sum_{p} \pi_{p l k m t}}{c a p_{m}} \leq n u m / I I_{m t}^{l k} \leq \frac{\sum_{p} \pi_{p l k m t}}{c a p_{m}}+1 \forall l, k, m, t \\
& \frac{\sum_{p} H_{p k r m t}}{c a p_{m}} \leq n u m / V_{m t}^{k r} \leq \frac{\sum_{p} H_{p k r m t}}{c a p_{m}}+1 \forall k, r, m, t \\
& \frac{\sum_{p} V T_{p K K^{\prime} m t}}{\operatorname{cap}_{m}} \leq n u m V_{m t}^{k k^{\prime}} \leq \frac{\sum_{p} V T_{p K K^{\prime} m t}}{\operatorname{cap}_{m}}+1 \forall k, k^{\prime}, m, t \\
& X_{p i j m t}, O_{p j l m t}, \pi_{p l k m t}, H_{p k r m t}, \mathrm{~S}_{k r p m t_{1}}, \mathrm{TR}_{k k^{\prime} m t_{1}}, \\
& \mathrm{~V} T_{p k k^{\prime} m t}, I_{p k k_{1} t}^{\prime}, I_{p k t}, l_{p k t} \geq 0 \\
& W_{i j t}, Y_{k l t}, \mathrm{G}_{k r t}, \text { tras }^{\prime}{ }_{j l t}, \text { tras }^{\prime \prime}{ }_{k k^{\prime} t} Z_{j t}, \mathrm{Vdc}_{k t}^{w}, F x_{i j m t}, F o_{j l m t}, F \pi_{l k m t} \text {, } \\
& F h_{k r m t}, F T_{k k^{\prime} m t} \in[0,1] \\
& \text { num } I_{m t}^{i j}, n u m / I_{m t}^{j l}, n u m / I I_{m t}^{l k}, n u m / V_{m t}^{k r}, n u m V_{m t}^{k k^{\prime}} \geq 0 \text { \& int }
\end{aligned}
$$

Objective function (1) shows total expenses of 3PL firm. The first part belongs to annual fixed cost of locating cross docks. The second part shows annual fixed cost of cross dock operation. The third part gives rental cost of the distribution center. The 4th, 5th, 6th, and 7th parts express transportation costs of 
various network layers. Part 8 states lateral transshipment costs among distribution centers. Part 9 shows upkeep costs of distribution center inventory. Part 10 shows shortage costs regarding not met demands. Objective function (2) describes sustainability social goals, the first part of which tries to increase fair distribution through minimizing the maximum shortage for each product in each period and customer region. The second part shows job opportunity increase considering the number of fixed jobs created by building cross dock and distribution center. The third part regards economic development of any area due to building cross dock and distribution center. Objective function (3) is related to environmental aspect of sustainability which tries to minimize fuel consumption considering the flow and the number of vehicles in the network which results in minimizing greenhouse gas emission (Bektas \& Laprte, 2011). Restrictions (4)-(11) state that a node cannot be allocated to a candidate node, unless it is built at a candidate location. Restrictions (12) and (13) insure that each producer and distribution center is allocated to one cross dock. Restriction (14) guarantees that each customer is allocated to one distribution center. Restriction (15) indicates that in case distribution center is selected and rented, the stored inventory should not exceed its capacity and in case it is not active, no inventory is stored. Restriction (16) states that the sent products from each producer should not exceed its production capacity. Restriction (17) shows shortage of each product for each region in any period. Restriction (18) insures that if the cross dock is built in the previous period; it will be fixed in upcoming periods. Restriction (19) shows that for each distribution center should be selected only one capacity in any period. Restrictions (20-24) suggest that the flow runs between two nodes when they are allocated. Restriction (25) indicates flow balance in cross dock. Restriction (26) shows the inventory of product P for a case where the difference of its production period $t_{1}$ from the period $t$ is less than $\operatorname{tmax}_{p}$ and is equal to the amount of received products in $t_{1}$ period from cross dock level (since storing is not possible in cross dock) plus the net amount of received products from other distribution centers in $t_{1}$ to $t$ range minus the amount of products delivered to customers in $t_{1}$ to $t$ range. Restriction (27) expresses that the total inventory stored of product $\mathrm{p}$ in period $\mathrm{t}$ is equal to the sum of inventories of the same product formed in different periods, t1. Restriction (28) states that total shipment flow from distribution center to customer in t period is equal to the sum of all flows from t1 to t periods. Restriction (29) suggests that total shipment flow from distribution center $k$ to distribution center $k$, in period $t$ is equal to sum of all flows in $\mathrm{t}_{1}$ - $\mathrm{t}$ period. Restriction (30) insures that the sum of all flows from all of the distribution centers will be equal to zero if the difference between period $t$ and production period of the product $t_{1}$ is greater than $t_{\max }$. Restriction (31) expresses that if the difference between period $t$ and production period of the product $t_{1}$ is greater than $t_{\operatorname{maxp}}$, no inventory is held, because the products will be corrupted and will be discarded. Restriction (32) shows that if distribution center is not selected in period $t$, the inventory of that center at the end of the period $t-1$ will be zero. Restrictions (33-37) indicate that vehicle will carry flow between arcs only when it is allocated to those arcs. Restrictions (38-42) are indicators of the number of vehicles required for each mode in each transportation arc considering the capacity of each vehicle mode Restrictions (43), (44), and (45) express systemic restrictions of the model and since the first part of objective function (1) and objective function (2) mentioned above are nonlinear, it is necessary linearize them to be able to solve them. In order to linearize the existing term in objective function (1), a new variable, $Z Z_{j t}=Z_{j t}\left(1-Z_{j t-1}\right)$ and restriction (46) is added to the model above.

$$
Z Z_{j t} \geq Z_{j t}+Z_{j t-1}-1
$$

To linearize objective function (2) another variable is defined $M M X=\max _{p r t}\left(d e_{p r t}-\sum_{k} \sum_{m} X_{p k r m t}\right)$ and restriction (47) is added to the model above.

$$
M M X \geq\left(d e_{p r t}-\sum_{k} \sum_{m} X_{p k r m t}\right)=\mathrm{ls}_{p r t} \quad \forall p, r, t
$$




\section{Solution approach}

In this section, possibilistic programming, which is a subset of fuzzy mathematical programming, is used to deal with the uncertainty considered in the model parameters. This is due to lack of knowledge regarding their real values. In order to create basic model of possibilistic chance constrained programming, based on Inuiguchi and Ramik (2000), Iwamura and Liu (1998) and Heilpern (1992), expected value operator is used to model inaccurate parameters of the objective function and credibility measure is used to model inaccurate parameters. Finally, the certain equivalent model of possibilistic programming of third party logistics network design by considering uncertain parameters of the model, total cost of the network and demand, is formulated as below. The first and the second objective functions are changed and restrictions (17) and (47) are converted to restrictions (50) and (51), respectively.

$$
\begin{aligned}
& \min Z_{1}:\left(\sum_{j} \sum_{t}\left[\frac{\left.F W_{j t}^{1}+2 F W_{j t}^{2}+F W_{j t}^{3}\right)}{4}\right]\left(Z_{j t}-Z Z_{j t}\right)+\sum_{j} \sum_{t}\left[\frac{F S_{j t}^{1}+2 F S_{j t}^{2}+F S_{j t}^{3}}{4}\right] Z_{j t}+\right. \\
& \sum_{k} \sum_{w} \sum_{t}\left[\frac{F K_{k w}^{1}+2 F K_{k w}^{2}+F K_{k w}^{3}}{4}\right] V d c_{k t}^{w}+\sum_{p} \sum_{i} \sum_{j} \sum_{l} \sum_{m} \sum_{t}\left[\frac{C_{i j m}^{1}+2 C_{i j m}^{2}+C_{i j m}^{3}}{4}\right] X_{p j i m t}+ \\
& \sum_{p} \sum_{j} \sum_{l} \sum_{m} \sum_{t} \theta\left[\frac{C T_{j m n}^{1}+2 C T_{j m n}^{2}+C T_{j m m}^{3}}{4}\right] O_{p j l h n t}+\sum_{p} \sum_{l} \sum_{k} \sum_{m} \sum_{t}\left[\frac{C P_{l m}^{1}+2 C P_{l m n}^{2}+C P_{l m m}^{3}}{4}\right] \pi_{p l h n t}+ \\
& \sum_{p} \sum_{k} \sum_{r} \sum_{m} \sum_{t}\left[\frac{C O_{k m m}^{1}+2 C O_{k m m}^{2}+C O_{k m m}^{3}}{4}\right] H_{p k m t}+ \\
& \sum_{p} \sum_{k^{\prime}} \sum_{k} \sum_{m} \sum_{t}\left[\frac{\operatorname{COST}_{k k^{\prime} m}^{1}+2 \operatorname{COST}_{k k^{\prime} m}^{2}+\operatorname{COST}_{k k^{\prime} m}^{3}}{4}\right] V T_{p k k^{\prime} m t}+ \\
& \sum_{p} \sum_{k} \sum_{r t} \sum_{t}\left[\frac{H C_{p l t}^{1}+2 H C_{p l t}^{2}+H C_{p l t}^{3}}{4}\right] I_{p l t t t}+\sum_{p} \sum_{r} \sum_{t}\left[\frac{H I s_{p t}^{1}+2 H l s_{p t}^{2}+H l s_{p t r}^{3}}{4}\right] l s_{p r t}
\end{aligned}
$$

$$
\begin{aligned}
& \max _{2}: \text { wp. } \frac{s i_{\max }^{m m x}-M M X}{s i_{\max }^{m m x}-s i_{\min }^{m m x}}+ \\
& w c . \frac{\sum_{j} \sum_{t} Z_{j t}\left[\frac{j c c_{j}^{1}+2 j c c_{j}^{2}+j c c_{j}^{3}}{4}\right] u p_{j}+\sum_{k} \sum_{w} \sum_{t} V d c_{k t}^{w}\left[\frac{j d c_{k w}^{1}+2 j d c_{k w}^{2}+j d c_{k w}^{3}}{4}\right] u p_{k}-s i_{\min }^{j c}}{s i_{\max }^{j c}-s i_{\min }^{j c}}+ \\
& \\
& w t . \frac{\sum_{j} \sum_{t} Z_{j t}\left[\frac{v p_{j}^{1}+2 v p_{j}^{2}+v p_{j}^{3}}{4}\right]\left(1-e d c_{j}\right)+\sum_{k} \sum_{w} \sum_{t} V d c_{k t}^{w}\left[\frac{v p_{k w}^{1}+2 v p_{k w}^{2}+v p_{k w}^{3}}{4}\right]\left(1-e d c_{k}\right)-s i_{\min }^{p t}}{s i_{\max }^{p t}-s i_{\min }^{p t}}
\end{aligned}
$$

subject to

Eqs. (4-46)

$$
\begin{array}{ll}
\sum_{k} \sum_{m} H_{p k r m t}+l s_{p r t} \geq(2-2 \alpha) d e_{p r t}^{2}+(2 \alpha-1) d e_{p r t}^{3} & \forall p, r, t \\
M M X \geq\left((2-2 \alpha) d e_{p r t}^{2}+(2 \alpha-1) d e_{p r t}^{3}-\sum_{k} \sum_{m} H_{p k r m t}\right) & \forall p, \mathrm{r}, t
\end{array}
$$


In the present paper, in order to calculate Pareto solutions demonstrating conflict of objective functions augmented epsilon constraint approach is used. Also, in order to solve the multi-objective problem and turning it into a single-objective model, a novel interactive fuzzy approach of Torabi and Hassini (2008) is utilized. These methods will be explained in the following.

\subsection{Augmented epsilon constraint method}

This method was presented by Mavrotas (2009) and the general equation of this method is shown in Eq. (52):

$$
\begin{aligned}
& \max \left(f_{1}(x)+\delta\left(\frac{s_{2}}{r_{2}}+\frac{s_{3}}{r_{3}}+\ldots+\frac{s_{p}}{r_{p}}\right)\right) \\
& f_{2}(x)-s_{2}=\varepsilon_{2} \\
& f_{3}(x)-s_{3}=\varepsilon_{3} \\
& \vdots \\
& f_{p}(x)-s_{p}=\varepsilon_{p} \\
& x \in S \text { and } s_{i} \in R^{+}
\end{aligned}
$$

where, $\delta$ is a small number between $10^{-6}$ and $10^{-3}, r_{1} r_{2}$ to $r_{p}$ are the ranges of their objective functions which are derived from payoff table (i.e. the optimum value of a objective function and its worst value). 1 - one of the objective functions, $f_{1}(x)$, is selected as the main objective function, in this case the first objective which is minimizing total costs of the system selected as the main objective 2- the problem is solved for one of the objective functions as a single objective each time and optimum values of each objective function and its worst values are obtained. In order to calculate worst values of the objective function, minimizing is changed to maximizing and vice versa. 3- The range between two optimum values of the secondary functions are divided into a certain number of sections and a table of values is obtained for $\varepsilon_{2}, \ldots, \varepsilon_{p}$. 4- The problem is solved each time for the main objective function using one of the $\varepsilon_{2}, \ldots, \varepsilon_{p}$ values. For problems having more than two objectives, $\varepsilon$ values should be determined for each objective function and solved the problem for all possible cases. 5- The obtained pareto answers are reported.

\subsection{The steps of fuzzy interactive approach proposed by Torabi and Hassini for solving a multi-objective certain model}

First step: We determine trapezoidal (or triangular) probability distribution functions appropriate for possibilistic parameters based on available historic data and experts'comments and setup of an initial multi-objective mix integer possibilistic linear programming for the mathematical model. Step two: We convert the objective function and possibilistic restrictions of the model into equivalent crisp equations and objectives using Nec index. Step three: In this setp, we determine positive ideal solution (PIS) and negative ideal solution (NIS) for each objective function. In order to obtain the positive ideal solution for each objective function, i.e. $\mathrm{Z}_{1}^{\mathrm{PIS}}, \mathrm{x}_{1}^{\mathrm{NIS}}$ and $\mathrm{Z}_{2}^{\mathrm{PIS}}, \mathrm{x}_{2}^{\mathrm{NIS}}$, it is necessary to solve equivalent certain models determined in the previous step separately for each objective function. Then, the negative ideal solution for each objective function is estimated as follows: 


$$
\begin{aligned}
& \mathrm{Z}_{1}^{\mathrm{NIS}}=\mathrm{Z}_{1}\left(\mathrm{x}_{2}^{\mathrm{PIS}}\right) \\
& \mathrm{Z}_{2}^{\mathrm{NIS}}=\mathrm{Z}_{2}\left(\mathrm{x}_{1}^{\mathrm{PIS}}\right)
\end{aligned}
$$

Step four: Now, we estimat a linear fuzzy membership function for each objective function based on Eq, (54) where $\mu_{\mathrm{i}}(\mathrm{x})$, represents satisfaction degree of $\mathrm{i}$ th objective function.

$$
\mu_{i}(x)= \begin{cases}1 & \text { if } Z_{i}<Z_{i}^{\text {PIS }} \\ \frac{Z_{i}^{N I S}-Z_{i}}{Z_{i}^{N I S}-Z_{i}^{P I S}} & \text { if } Z_{i}^{\text {NIS }} \leq Z_{i} \leq Z_{i}^{\text {PIS }} \\ 0 & \text { if } Z_{i}>Z_{i}^{N I S}\end{cases}
$$

Step five: In this step, we convert the certain multi-objective integer linear model into a single-objective model using merger function proposed by Torabi and Hassini (2008) as follows.

$$
\max \lambda(\mathrm{x})=\psi \lambda_{0}+(1-\psi) \sum_{\mathrm{i}} \sigma_{\mathrm{i}} \mu_{\mathrm{i}}(\mathrm{x})
$$

subject to

$$
\begin{aligned}
& \lambda_{0} \leq \mu_{\mathrm{i}}(\mathrm{x}), \\
& \mathrm{x} \in \mathrm{F}, \lambda_{0} \text { and } \lambda \in[0,1]
\end{aligned}
$$

where $F$ indicates feasible region formed based on crisp (non-fuzzy) restrictions of the equivalent secondary model, $\sigma_{i}$ and $\Psi$ represent degree of importance (priority) of ith objective function and the coefficient of compensation, respectively. $\sigma_{i}$ values are based on decision maker's preferences and are determined so that $\sum_{\mathrm{i}} \sigma_{\mathrm{i}}=1$ and $\sigma_{\mathrm{i}} \geq 0$. It must be noted that the optimum value of variable $\lambda_{0}=$ $\min _{\mathrm{i}}\left\{\mu_{\mathrm{i}}(\mathrm{x})\right\}$ indicates the minimum satisfaction degree of objective functions. Therefore, merging function of Torabi and Hassini (2008) is used to find an intermediate (adaptive) value between this minimizing operator $\left(\lambda_{0}=\min _{\mathrm{i}}\left\{\mu_{\mathrm{i}}(\mathrm{x})\right\}\right)$ and weighted sum of objective functions $\left(\sum_{\mathrm{i}} \sigma_{\mathrm{i}} \mu_{\mathrm{i}}(\mathrm{x})\right)$ according to the determined value for correction coefficient. In other words, the decision maker can determine the tradeoff of his/her desired solutions by adjusting the values of $\sigma_{i}$ and $\Psi$ based on his/her assessment, preferences, and priorities. Step six: In this setp, we determine corrected coefficient $(\Psi)$ and relative importance of fuzzy objectives $\left(\sigma_{i}\right)$, and solve the integer linear programming certain single-objective model. If the resulted answers are satisfactory for the decision maker, we stop the solution, otherwise we try again to find a new answer by readjusting the values of $\sigma_{i}$ and $\Psi$ and go to step five. It should be mentioned that choosing higher values for $\Psi$ means more focus to find a greater value for lower bound of satisfaction degree of objectives $\left(\lambda_{0}\right)$, thus the final solution will be more balanced. On the other hand, choosing a lower value for $\Psi$ means more focus to obtain a solution with higher satisfaction degree for some objectives of the problem while ignoring some others. Therefore, the final solution in such case will be a more imbalanced one.

\section{Computational results}

In this section, in order to validate the performance of the proposed model, a numerical example is generated, randomly by considering parameter ranges and the resulted problem is solved using GAMS software in a Corei5 computer with 4 gigabyte RAM. For this sample problem, parameters are considered as Table 1.

Then, the conflict of objective functions is demonstrated using augmented epsilon constraint method. Also, various sensitivity analyses using multi-objective method of Torabi and Hassini are presented. 
Table 1

Input parameter values for solving problem

\begin{tabular}{ll}
\hline Parameters & Parameters \\
\hline$v_{i j}{ }^{m}=$ uniform $(20,100) ;$ & $w^{m}=$ uniform $(900,1000) ;$ \\
$c a p_{m}=$ uniform $(1000,1500) ;$ & $F K_{k w}{ }^{1}=0.9 \times F K_{k w}{ }^{2}$ \\
$N^{m}=$ uniform $(30,50) ;$ & $F K_{b w .}{ }^{2}=$ uniform $(10,15) ;$ \\
$K^{m}=$ uniform $(0.2,0.5) ;$ & $F K_{k w}{ }^{3}=1.1 \times F K_{k w}{ }^{2}$ \\
$\Gamma^{m}=$ uniform $(0.002,0.005) ;$ & $C T_{j l m}{ }^{1}=0.9 \times C T_{j l m}{ }^{2}$ \\
$v d^{m}=$ uniform $(2,5) ;$ & $C T_{i l m}{ }^{2}=(0,200)$ \\
$l a_{k w}=$ uniform $(800,900) ;$ & $C T_{j l m}{ }^{3}=1.1 \times C T_{j l m}{ }^{2}$ \\
$\alpha_{i j}^{m}=$ uniform $(0.5,0.9) ;$ & $d e_{p r t}{ }^{1}=0.8 \times d e_{p r t}{ }^{2}$ \\
$F W_{j t}{ }^{1}=0.9 \times F W_{j t}{ }^{2}$ & $d e_{p r t}{ }^{2}=10$ \\
$F W_{j t}{ }^{2}=$ uniform $(100,150) ;$ & $d e_{p r t}{ }^{3}=1.2 \times d e_{p r t}{ }^{2}$ \\
$F W_{j t}{ }^{3}=1.1 \times F W_{j t}{ }^{2}$ & $\theta=0.1$ \\
$t \max _{p}=2$ & $e d c_{j}=$ uniform $(0.2,0.4)$ \\
$\sigma_{1}=0.2$ & $\sigma_{3}=0.5$ \\
$\sigma_{2}=0.3$ & $\psi=0.5$ \\
\hline
\end{tabular}

\subsection{Conflict of objective functions}

As we know, there is no unique answer in epsilon constraint method which can optimize all objectives and due to the conflict between objectives, as one gets more desirable values, the others fall into more undesirable values. As it can be seen in Fig. 2, objective function of minimizing network costs $\left(\mathrm{Z}_{1}\right)$ and objective function of maximizing social aspects of the network $\left(\mathrm{Z}_{2}\right)$ are in contrast with each other. To justify the conflict between system costs and social aspects of the network, it can be stated that the objective function of costs tends to decrease building cross docks and distribution center selections which results in decreasing social objective function, because social objective function tends to maximize job opportunity and economic development through building more cross docks and distribution centers. Fig. 2 illustrates the relationship between these objective functions.

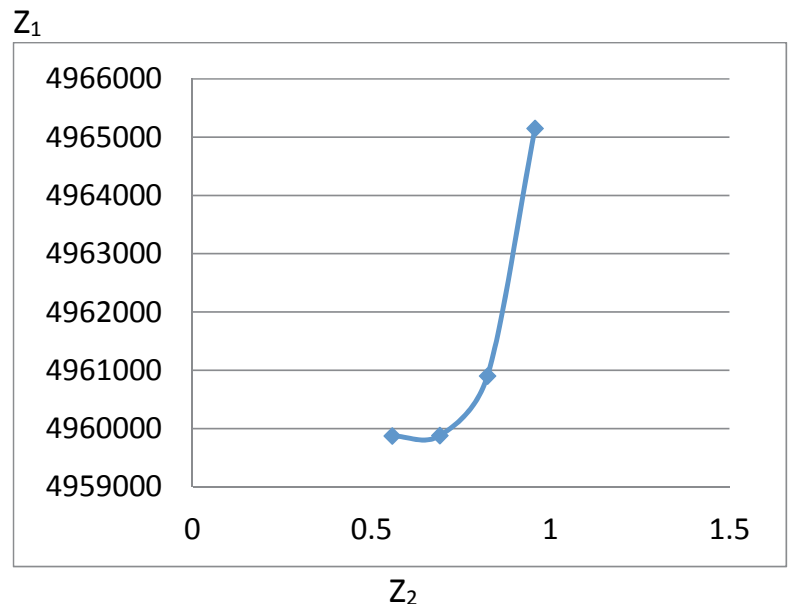

Fig. 2. comparison between objective functions 1 and 2 (pareto boundary)

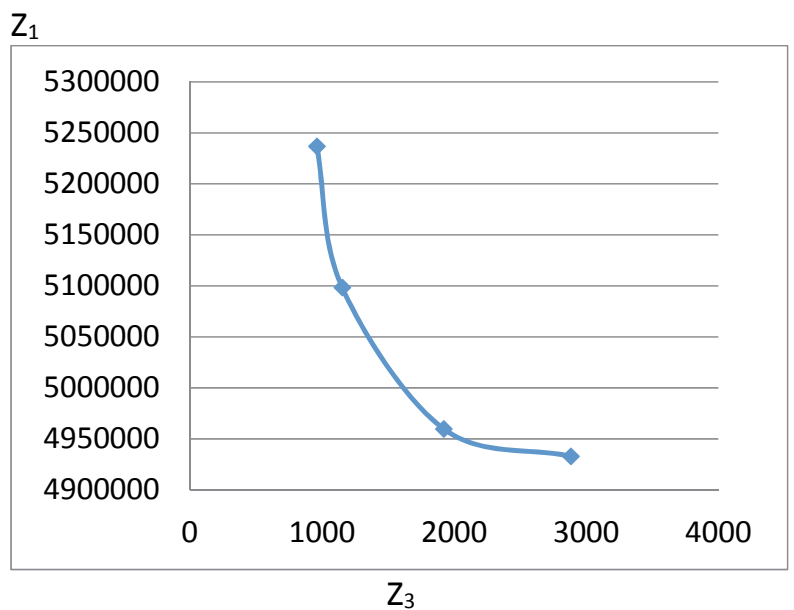

Fig. 3. Comparison of objective functions 1 and 3 (pareto boundary) 
Comparing objective function of system costs with objective function of environment is important, because the model tends to choose cheap transportation modes to reduce costs which will increase the emission of greenhouse gases. Fig. 3 shows that reducing costs, causes greenhouse gas emissions to increase.

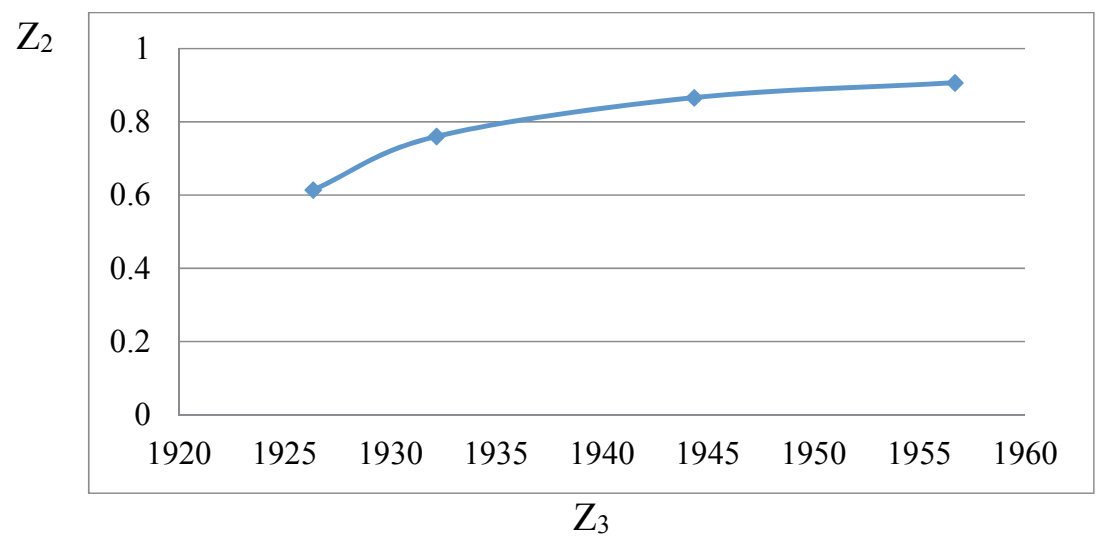

Fig. 4. comparison of objective functions 2 and 3 (pareto boundary)

As it can be seen in Fig. 4, the objective function of minimizing greenhouse gas emissions is in contrast with the objective function of maximizing social aspects. Because the environmental objective function desires no flow in the network so that no vehicle is used and greenhouse gas emissions reaches zero, which causes social aspect of the network to decrease. Because, social objective function tries to minimize the maximum shortage in the network via forming flows which leads to increasing greenhouse gas emissions.

\subsection{Sensitivity analysis}

Now, several sensitivity analyses are executed on the aforementioned problem to present a managerial outlook and results are reported.

- Sensitivity analysis results from changing maximum perishable time periods parameter $T \max _{p}$

For this purpose, the number of perishable time period is increased to 5 periods and is then compared to the basic perishable time period of the problem which is 2 . As it can be seen in Fig. 5, increasing the number of perishable time periods reduces system costs, since as $T \max _{p}$ value increases more feasible solution can emerge and also it will be possible to hold inventory for more periods. This can help reducing problem costs, since it allows for more goods to be received in a period and held for more periods.

$\mathrm{Z}_{1}$

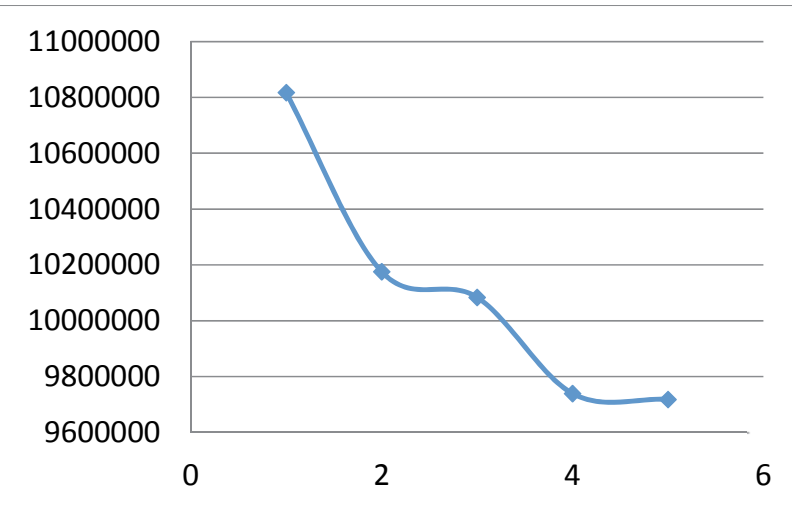

The number of perishable time period

Fig. 5. Sensitivity of first objective function $\left(Z_{1}\right)$ versus changes in $T \max _{p}$

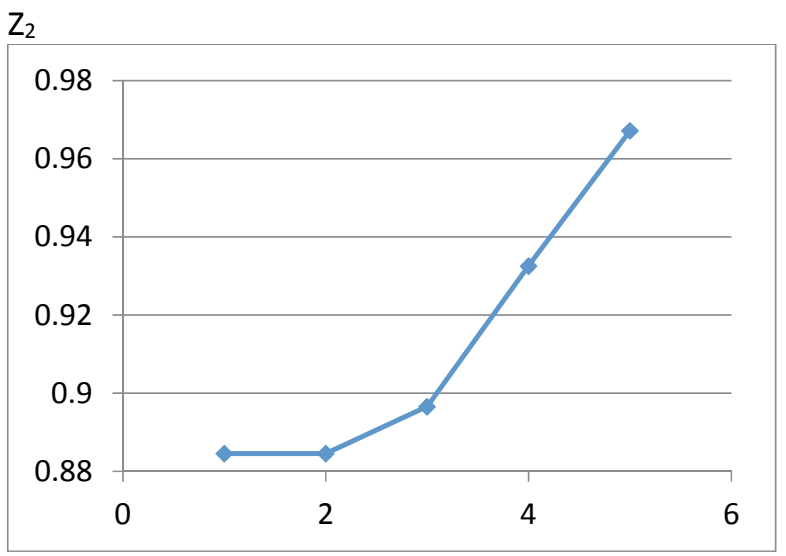

The number of perishable time period

Fig. 6. sensitivity of second objective function $\left(\mathrm{Z}_{2}\right)$ versus changes in $T \max _{p}$ 
As it can be observed in Fig. 6, the value of the second objective function increases as the number of perishable time periods increases. Since, as $T \max _{p}$ increases it allows for more goods to be received in a period and held for a longer time which makes it easier to control shortage. Also, the results from sensitivity analysis of cost parameter is the third objective function $\left(Z_{3}\right)$ shown in Fig. 7. As mentioned before, increasing the number of perishable time period causes products to be transshipped from producer levels to distribution centers in less amount of time but more amount which leads to less usage of vehicles which causes the third objective function value to decrease.

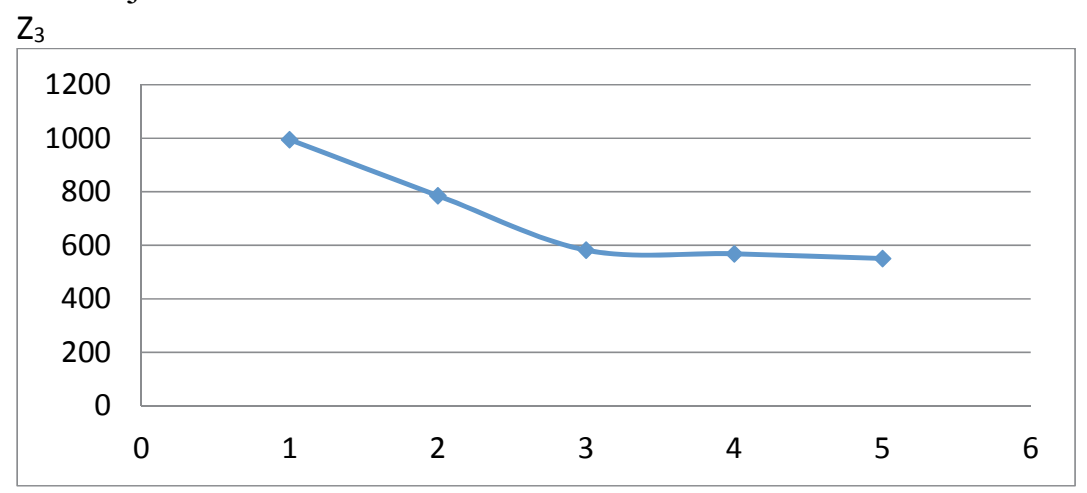

The number of perishable time period

Fig. 7. sensitivity of third objective function $Z_{3}$ versus changes in $T \max _{p}$

- Sensitivity analysis results of elimination scenario of lateral transshipment between cross docks and distribution centers

As mentioned before, lateral transshipment has always been considered as a way to reduce costs and compensate shortage when access to some nods is not possible due to coverage radius. In this problem, lateral transshipment exists between cross docks and distribution centers which benefits from discount at cross dock level. In this paper, during a scenario, the main problem is compared with a case where there is no lateral transshipment, in order to study the influence of lateral transshipment in problem optimization. For this purpose, the problem is solved considering lateral transshipment and then solved again considering Eq. (56).

$$
\sum_{j} \sum_{l} \sum_{t} \operatorname{tras}^{\prime}{ }_{j l t}+\sum_{k} \sum_{k^{\prime}} \sum_{t} \operatorname{tras}^{\prime \prime}{ }_{k k^{\prime} t}=0
$$

The results after solving the problem with two scenarios are summarized in Table 2 as follows,

Table 2

The results by considering two scenarios

\begin{tabular}{ccccc}
\hline & Without lateral transshipment & With lateral transshipment & different & Gap\% \\
\hline $\mathrm{Z}_{1}$ & 10208952.72 & 9458169 & 750783.7 & 7.937939362 \\
$\mathrm{z}_{2}$ & 0.87980986 & 0.89714623 & .01904761 & -2.123134264 \\
$\mathrm{Z}_{3}$ & 690.0904063 & 648.4821 & 41.60831 & 6.416261343 \\
\hline
\end{tabular}

As it can be seen from the results of Table 2, considering lateral transshipment improves all objectives; it improves cost and environmental objective functions and also yields a higher social index. 


\section{Conclusion and future study}

The presented model is a new model for multi-objective linear mix integer programing in dynamic conditions for logistics sustainable network design of third party providers. This model is a three-objective model, which, in addition to cost objective function, considers sustainability issues as extra objectives in the model. These objectives include environmental aspects such as greenhouse gas emissions due to various modes of transportation as well as social aspects such as fair accessibility of products for every customer region in each time period, increasing job opportunities in cross docks and distribution centers according to unemployment rate of each region, and increasing economic development balance through focusing more on regions with lower development. This model is solved using augmented epsilon constraint method in order to create Pareto solution. The results of this method have indicated conflicts among the three objective functions; in other words, as one of the objectives, such as social objective, improves, cost and environment objectives tend to reach undesirable values. According to these conflicts among objective functions, it can be concluded that the firm must spend more money than the case where only economic aspect is considered, in order to maintain environmental and social aspects. Sensitivity analysis on input parameter of maximum perishable time period has shown that increasing this parameter could improve all three objective functions. Moreover, considering lateral transshipment among facilities of a level can improve sustainability indices of the problem which indicates the necessity of such policy in improving network sustainability. The following suggestions are presented for future studies:

1. Implementation of the model and utilizing it in a real case,

2. Using meta-heuristic algorithms for solving big size problems,

3. Utilizing benefits of robust optimization to oppose uncertainties existing in the model.

\section{Acknowledgement}

The authors would like to thank the anonymous referees for constructive comments on earlier version of this paper.

\section{References}

Boyson, S., Corsi, T., Dresner, M., \& Rabinovich, E. (1999). Managing effective third party logistics relationships: what does it take?. Journal of Business Logistics, 20(1), 73-100

Pishvaee, M. S., Razmi, J., \& Torabi, S. A. (2014). An accelerated Benders decomposition algorithm for sustainable supply chain network design under uncertainty: A case study of medical needle and syringe supply chain. Transportation Research Part E: Logistics and Transportation Review, 67, 1438.

Mula, J., Poler, R., \& Garcia, J. P. (2006). MRP with flexible constraints: A fuzzy mathematical programming approach. Fuzzy Sets and Systems, 157(1), 74-97.

Ko, H. J., \& Evans, G. W. (2007). A genetic algorithm-based heuristic for the dynamic integrated forward/reverse logistics network for 3PLs. Computers \& Operations Research, 34(2), 346-366.

Zhang, Y., Xie, L., Hang, W., \& Cui, X. (2007, August). A robust model for 3PLS to design a remanufacturing logistics network under the uncertain environment. In Automation and Logistics, 2007 IEEE International Conference on (pp. 367-372). IEEE.

Mallidis, I., Dekker, R., \& Vlachos, D. (2012). The impact of greening on supply chain design and cost: a case for a developing region. Journal of Transport Geography, 22, 118-128.

Ghaffari-Nasab, N., Ghazanfari, M., \& Teimoury, E. (2015). Hub-and-spoke logistics network design for third party logistics service providers. International Journal of Management Science and Engineering Management, (ahead-of-print), 1-13.

Dehghanian, F., \& Mansour, S. (2009). Designing sustainable recovery network of end-of-life products using genetic algorithm. Resources, Conservation and Recycling, 53(10), 559-570. 
Devika, K., Jafarian, A., \& Nourbakhsh, V. (2014). Designing a sustainable closed-loop supply chain network based on triple bottom line approach: A comparison of metaheuristics hybridization techniques. European Journal of Operational Research, 235(3), 594-615.

Ramezani, M., Kimiagari, A. M., Karimi, B., \& Hejazi, T. H. (2014). Closed-loop supply chain network design under a fuzzy environment. Knowledge-Based Systems, 59, 108-120.

Torabi, S. A., \& Moghaddam, M. (2012). Multi-site integrated production-distribution planning with trans-shipment: a fuzzy goal programming approach. International Journal of Production Research, 50(6), 1726-1748.

Bektaş, T., \& Laporte, G. (2011). The pollution-routing problem. Transportation Research Part B: Methodological, 45(8), 1232-1250.

Inuiguchi, M., \& Ramık, J. (2000). Possibilistic linear programming: a brief review of fuzzy mathematical programming and a comparison with stochastic programming in portfolio selection problem. Fuzzy sets and systems, 111(1), 3-28.

Iwamura, K., \& Liu, B. (1998). Chance constrained integer programming models for capital budgeting in fuzzy environments. Journal of the Operational Research Society,49(8), 854-860.

Heilpern, S. (1992). The expected value of a fuzzy number. Fuzzy sets and Systems, 47(1), 81-86.

Mavrotas, G. (2009). Effective implementation of the $\varepsilon$-constraint method in multi-objective mathematical programming problems. Applied mathematics and computation, 213(2), 455-465.

Torabi, S. A., \& Hassini, E. (2008). An interactive possibilistic programming approach for multiple objective supply chain master planning. Fuzzy Sets and Systems, 159(2), 193-214. 